\title{
Allgemeingültige Sätze - eine Herausforderung für die Prototypensemantik
}

\section{Ein erster Überblick}

Allgemeingültige oder generische Sätze bilden einen notorischen Problemkomplex für jede Art von Semantik, gleichgültig von welcher theoretischen Provenienz. ${ }^{1}$

Wenn wir von generischen Sätzen sprechen, so ist das übrigens eine verkürzte Redeweise, und wir sollten eigentlich von Sätzen in generischer Lesart reden, da solche Sätze zumindest in der geschriebenen Sprache auch eine nicht-generische Lesart haben. In gesprochener Sprache kann hier durch Betonung oft Eindeutigkeit geschaffen werden. Der Witz von dem Rabbiner, der auf ein Propagandaplakat mit der Inschrift: Ein Deutscher lügt nicht! mit: Das is aber a mieses Perzent fir so a großes Volk! reagiert, ist auf die schriftliche Version des Slogans angewiesen. ${ }^{2}$

Solche generischen Sätze sind z.B.:

(1a) Eine Schwalbe macht noch keinen Sommer.

(1b) Der Schwabe ist fleißig.

(1c) Katzen würden Whiskas kaufen.

(1d) Die Mongolen waren geschickte Reiter.

(1e) Löwen haben eine Mähne.

(1f) Der Läufer zieht diagonal. (beim Schach)

Sie haben alle, ungeachtet der verschiedenen Form der Nominalphrasen, eine generische Interpretation, können also alle über einen Leisten geschlagen werden. Das wirft zwei Hauptprobleme auf, nämlich e $r$ s $t$ e $n$ s: Wie sieht der gemeinsame Leisten aus? und z w e i t e n s: Wie kommen diese doch recht verschiedenen Sätze auf

1 Dieser Beitrag entstand aus meiner Arbeit an der „Grammatik des heutigen Deutsch", die in den letzten Jahren am IdS erarbeitet wurde. Für Diskussionen früherer Fassungen, die zu wesentlichen Verbesserungen führten, danke ich meinen Kolleginnen und Kollegen Ursula Brauße, Ursula Hoberg, Renate Pasch, Ludger Hoffmann, Bruno Strecker, Klaus Vorderwülbecke und ganz besonders Gisela Zifonun und Helmut Frosch.

2 Dietrich Hartmann hat mich am Rande der Tagung darauf hingewiesen, $\mathrm{da} B$ in zahlreichen süddeutschen Dialekten zwischen dem auch generisch verwendbaren unbestimmten Artikel und einem Anzahlquantor für eins differenziert wird, so z.B. auch in Mannheim: ̈̈ Schwalb (Artikel) versus ää Schwalb (numerischer Quantor). 
diesen einen Leisten? Was die erste Frage betrifft, so hat man immer schon gewußt, daß die Bedeutung von Sätzen in generischer Interpretation irgendwie so ähnlich wie die von Allsätzen beschrieben werden muB, jedoch mit dem kleinen Unterschied, daß sie „schwächer” ist, daß sie als Radio-Eriwan-Allsatz formuliert werden kann, z.B. für (1e): „Alle Löwen haben im Prinzip eine Mähne." Weniger klar war jedoch, wie der kleine Unterschied beschrieben werden kann. ${ }^{3}$

Hier scheint die Prototypensemantik eine attraktive Antwort anbieten zu können. Ich zitiere eine gängige Definition des Prototyps:

"A prototype is a (mental representation) (possibly one quite rich in specific detail) of a) typical instance of a Category, such that entities get assimilated to the category on the basis of perceived similarity to the prototype...n 4

Zunächst scheint klar, daß der Zusatz von Taylor nötig ist, denn wäre der Prototyp ein E x e m p l a r, dann wäre der erste Prototypensemantiker jener Professor in der Akademie von Lagado in Balnibarbi gewesen, von dem Gulliver berichtet, er wolle die Wörter abschaffen:

„... since words are only names for things, it would be more convenient for all men to carry about them such things as were necessary to express the particular business they are to discourse on. And this invention would certainly have taken place, to the great ease as well as health of the subject, if the women in conjunction with the vulgar and illiterate had not threatened to raise a rebellion, unless they might be allowed the liberty to speak with their tongues, after the inanners of their forefathers: such constant irreconcileable enemies to science are the common people. However, many of the most learned and wise adhere to the new scheme of expressing themselves by things; which hath only this inconvenience attending it; that if a man's business be very great, and of various kinds, he must be obliged in proportion to carry a greater bundle of things upon his back, unless he can afford one or two strong servants to attend him. ${ }^{n 5}$

Aber von dem mentalistisch erweiterten Konzept aus scheint es verführerisch, einen Satz wie (1b) so zu analysieren: Der prototypische Schwabe ist fleißig (und alle Schwaben, die ihm hinreichend ähneln, sind es ebenfalls). Das klingt auf den ersten Blick, wenn man von dem in Prototyplerkreisen geradezu stigmatisierten "hinreichend” absieht, ganz ein-

3 Wesentliche Beiträge dazu wurden im letzten Jahrzehnt im Rahmen der logischen Semantik und der künstlichen Intelligenz gemacht, man sehe dazu z.B. die Beiträge in Krifka (1988).

4 Taylor (1989, S. 529), ohne die eingeklammerte Passage schon bei Langacker (1987, S. 371).

5 Swift (1726), zitiert nach der Wordsworth Classics Ausgabe, (1992, S. $197 / 198$ ). 
leuchtend und auch schön einfach, und vielleicht braucht man noch nicht einmal den Zusatz in der Klammer. Und (le) würde dann analysiert als: Ein prototypischer Löwe hat eine Mähne.

Fügen wir noch das Beispiel:

\section{(1g) Ein Löwe bringt lebende Junge zur Welt.}

hinzu, so wird das schon problematischer, denn dann haben wir einen prototypischen Löwen, der nach (1e) eine Mähne hat, und der nach (1g) lebende Junge zur Welt bringt. Das erinnert fatal an das existentiell generische Schwein bei David Lewis:

"In the dark ages of logic, a story something like this was told. The phrase 'some pig' names a strange thing we may call the existentially generic pig which has just those properties that some pig has. Since some pig is male, some pig (a different one) is female, some pig is pink (all over), and some pig is grey (all over), the existentially generic pig is simultaneously male, female, pink, and grey." 6

Eine Lösung, die sich anbietet, wäre, bei Bezeichnungen natürlicher Arten wie Löwe einen männlichen und einen weiblichen Prototyp zu haben, um sich dann bei Sätzen in generischer Interpretation jeweils auf die eine, den anderen oder beide zu beziehen. Aber es liegt auf der Hand, daß diese Lösung bei komplexeren Kategorien, die nicht dem „basic level” zuzurechnen sind, wie z.B. Raubkatze, Säugetier etc. zu einer Bevölkerungsexplosion im Lande Prototypia führen würde, die die Anziehungskraft einer solchen Theorieskizze ziemlich vermindern dürfte. ${ }^{7}$

Von soviel Skepsis angekränkelt will ich im folgenden versuchen, zunächst zu zeigen, daB es sich bei den sogenannten generischen Sätzen keinesfalls um ein einheitliches Phänomen handelt, und daß die oben angesprochenen Interpretationsprobleme eigentlich nur bei e i n e m Typ; auftauchen. Danach versuche ich eine semantische Analyse im Rahmen einer mit klassischen Mitteln arbeitenden wahrheitskonditionalen Semantik (die Frage nach dem einheitlichen Leisten!), und ich versuche dann zu zeigen, wie man mit semantischen und pragmatischen Mitteln von der „normalen” Bedeutung dieser Sätze zu ihrer generischen Lesart gelangen kann (die Frage, wie die verschiedenen Stiefel auf den eirien Leisten passen).

6 Lewis (1972, S.203).

7 Der Ausweg von Lewis (1972), der darin besteht, überzugehen auf 'characters', verstanden als Mengen von Eigenschaften, also abstrakte Konstrukte, scheint mir für Prototypensemantiker nicht gangbar, da dann die realistisch-mentalistische Tōnung diese Ansatzes dahin wäre. 
1.1 Versuch einer Typologisierung

GATTUNGSPRÄDIKATION

Betrachten wir die Sätze:

(2a) Der Löwe ist eine Raubkatze.

(2b) Philipp Drais erfand das Fahrrad.

(2c) Das Mammut ist ausgestorben.

Man sieht, daß es hier um Prädikate geht, die auf einzelne Individuen nicht vernünftig angewendet werden können, sondern der ganzen Gattung zugesprochen werden müssen.

\section{ABGELEITETE GATTUNGSPRÄDIKATION}

Im Kontrast dazu stehen die Sätze

(3a) Ein Löwe hat eine Mähne.

(3b) Katzen miauen.

wo über ein einzelnes Individuum prädiziert wird, das als typischer Vertreter der Gattung gilt. ${ }^{8}$ Dieser Unterschied korreliert nicht mit der Unterscheidung NP mit bestimmtem versus NP mit unbestimmtem Artikel. Das zeigen:

(4a) Philipp Drais erfand ein Fahrrad. (Nämlich die Draisine.) $)^{9}$

(4b) Der Löwe hat eine Mähne.

In (4a) wird durch das Verb erfinden eine Interpretation von ein Fahrrad als Subgattung erzwungen, und in (4b) bezieht der Löwe sich nicht auf die Gattung. Bevor ich der Idee, daß unser Problem etwas mit Gattungsbezeichnungen zu tun hat, weiter nachgehe, will ich zunächst noch die folgenden Verwendungsweisen ausgrenzen:

\section{LEO IN FABULA}

Ebenfalls einen Sondertyp bilden Sätze, in denen es nicht um reale Individuen geht, sondern um Fabelwesen und ontologisch ähnlich dubiose Gesellen:

(5a) Der Löwe ist der König der Tiere.

(5b) * Die Löwen sind die Könige der Tiere.

(Keine Paraphrase von (5a)!)

8 Die Bezeichnung 'abgeleitete Gattungsprädikation' ('derived kind predication') stammt aus Link (1988, S. 314)

9 Der Satz geht also, entgegen der in Eisenberg (1986, S. 156) vertretenen Meinung. 
(5c) Löwen sind die Könige der Tiere. (Siehe (5b)!)

(5d) ?Ein Löwe ist der König der Tiere. (Eher: Der König der Tiere ist ein Löwe.)

(5e) *Alle Löwen sind König/Könige der Tiere.

(5f) * Jeder Löwe ist König der Tiere.

Hier geht es bei genauerer Betrachtung gar nicht um generische Interpretation, sondern um ein Fabelwesen, dem etwas zugeschrieben wird, Ito in $f$ abula. Dessen naher Verwandter $\mathbf{X}$ to th tolokul begegnet uns in:

(6a) Der Löwe ist das Wappentier von Bayern.

(6b) Im Schilde wird geführt der Leu von England wie vom Löwenbräu. ${ }^{10}$

Er ist ein munterer Geselle, der mehr Funktionen wahrnehmen kann, als ein Großbankier Aufsichtsratsmandate - und das alles auch noch gleichzeitig! Außerdem gibt es noch Yro hikrogl!phikıs

(6c) Der Löwe ist die Hieroglyphe, die dem l entspricht.

Für unser eigentliches Problem können wir diese drei getrost sich selbst überlassen.

LAW AND ORDER

Einen weiteren Typ illustrieren:

(7a) Der Läufer zieht diagonal.

(7b) Die Läufer ziehen diagonal.

(7c) Läufer ziehen diagonal.

(7d) Ein Läufer zieht diagonal.

(7e) ?Alle Läufer ziehen diagonal.

(7f) ?Jeder Läufer zieht diagonal.

Hier geht es um Sätze, die auf Gesetzmäßigkeiten oder Regeln rekurrieren, deren Allgemeingültigkeit außer Frage steht - wenn ein Läufer anders als diagonal zieht, bewegen wir uns außerhalb des Schachspiels; deshalb sind auch die Fassungen (7e,f) mit Quantoren Verstöße gegen die Maxime: „Mach Deinen Gesprächsbeitrag nicht informativer als nötig!” - daher die Fragezeichen. Damit verwandt sind „ewige Sätze”, wie:

(7g) Zwei mal zwei ist vier.

die wir hier ebensowenig ausführlich behandeln wollen wie temporale generische Interpretation von Beispielen wie:

10 Roth (1956, S. 27). 
(7h) An der Bergstraße ist es warm.

oder taxonomische Sätze ${ }^{11}$ wie:

(7i) Pferde sind Unpaarhufer.

\section{GATTUNGEN UND GATTUNGSPRÄDIKATION}

Damit komme ich zu der Frage zurück, inwieweit und in welcher Weise Gattungsbezeichnungen für generische Interpretationen konstitutiv sind. Betrachten wir dazu:

(8a) Der Saurier ist ausgestorben.

(8b) Die Saurier sind ausgestorben.

(8c) Saurier sind ausgestorben. (mehrere Subgattungen)

(8d) Ein Saurier ist ausgestorben. (eine Subgattung)

(8e) Alle Saurier sind ausgestorben. (alle Subgattungen)

(8f) * Jeder Saurier ist augestorben.

Hier liegt ein n i cht-dist $\mathrm{r}$ i b u t ives Prädikat vor, das außerdem nur über Gattungen prädiziert werden darf - das zeigt die Unmöglichkeit von Sätzen wie:

(8g) *Die drei Musketiere sind ausgestorben.

Wir haben also zwei BEDEUTUNGSPOSTULATE für aussterben:

I. GATTUNGSPRÄDIKAT: $\forall(\mathrm{x})(\mathfrak{A}(\mathrm{x}) \rightarrow \mathfrak{( x )})$

II. NICHT-DISTRIBUTIVITÄT: $\forall(\mathrm{x})\left(\forall(\mathrm{y})\left(\neg\left(\left(\boldsymbol{A}(\mathrm{x}) \wedge(\mathrm{x}) \Delta_{\pi} \mathrm{y}\right)\right.\right.\right.$ $\rightarrow \mathbf{A}(\mathrm{y})))$ )

( $\forall$ ist der Allquantor, $\exists$ der Existenzquantor, $\Delta_{\pi}$ ist die "enthält"-Relation für Kollektive ${ }^{12}$, die als Denotate von Plural-Nominalphrasen dienen.)

11 Link (1988, S. 315) spricht von 'taxonomic generics' unter Berufung auf Krifka (1987).

12 In der Behandlung der Semantik von Plural- und Substanz-Nominalphrasen folge ich der in Link (1983) entwickelten Theorie, die in Link (1991) und Krifka (1991) nochmals ausführlich dargestellt ist. Für unseren Zusammenhang ist vor allem wichtig, daß die Denotate pluralischer Nominalphrasen Kollektive oder Summenobjekte sind, für die eine „enthält"-Relation definiert ist. Der Denotatbereich einer Plural-NP ist dann ein Summenhalbverband von solchen Kollektiven. 
Das zweite Postulat besagt einfach, daß die Eigenschaft, ausgestorben zu sein, wenn sie einem Kollektiv zugeschrieben wird, $\mathrm{n} \mathrm{i} \mathrm{cht}$ auf dessen Teile und insbesondere $\mathrm{n} i \mathrm{c} \mathrm{h} \mathrm{t}$ auf die einzelnen Individuen des Kollektivs zutrifft. Das erste, in unserem jetzigen Zusammenhang wichtigere Postulat besagt, daB wenn auf etwas die Eigenschaft zutrifft, ausgestorben zu sein, dieses eine Gattung sein muB. Dadurch wird eine Interpretation der Nominalphrasen als Gattungsbezeichnung erzwungen. Da das in (8f) wegen des distributiven Quantors jeder nicht geht, ist der Satz nicht möglich (für manche Sprecher geht er in einer Subgattungslesart.).

Auch bei diesen Beispielen stellt sich das oben skizzierte Problem einer Interpretation als abgeschwächter Allsatz nicht.

\section{GATTUNGEN UND DISTRIBUTIVE PRÄDIKATION - DAS EI- GENTLICHE PROBLEM}

Betrachten wir nun Beispiele, in denen ein dis t r i b u t i v e s Prädikat mit einer Gattungsbezeichnung zusammentrifft, d.h. ein Prädikat, das sich von Kollektiven, auf die es zutrifft, auf a ll e Teile einschließlich der Einzelindividuen überträgt.

(9a) Der Löwe hat eine Mähne.

(9b) Die Löwen haben eine Mähne/Mähnen.

(9c) Löwen haben eine Mähne.

(9d) Ein Löwe hat eine Mähne.

(9e) Alle Löwen haben eine Mähne.

(9f) Jeder Löwe hat eine Mähne.

Offenbar sind $(9 a-$ d) nun endlich Beispiele, deren Wahrheitsbedingungen in der generischen Lesart im oben ausgeführten Sinne problematisch sind. Für distributive Prädikate, z.B. für hat eine Mähne, gibt es ein BEDEUTUNGSPOSTULAT:

DISTRIBUTIVITÄT: $\forall(\mathrm{x})\left(\forall(\mathrm{y})\left(\left(\boldsymbol{A}(\mathrm{x}) \wedge \mathrm{x} \Delta_{\pi} \mathrm{y}\right) \rightarrow \operatorname{su}(\mathrm{y})\right)\right)$

Dies stellt sicher, daß die entsprechende Eigenschaft, wenn das Prädikat auf ein Pluralobjekt, eine Summe, zutrifft, auf die darin enthaltenen Objekte "heruntergerechnet" werden darf.

Damit haben wir das Interpretationsproblem bei allgemeingültigen Sätzen auf diesen Satztyp eingeschränkt und können uns jetzt auf unsere zwei Hauptprobleme konzentrieren, und wir bleiben zunächst bei der Frage nach dem einheitlichen Leisten, der generischen Interpretation. 


\section{Semantik für generische Sätze}

In der Literatur besteht weitgehend Einigkeit darüber, daß Sätze wie $(1 \mathrm{a}-\mathbf{f})$ als eine abgeschwächte Version von Allaussagen zu verstehen sind, denn man wird ja die Existenz eines unfleißigen oder gar faulen Schwaben nicht als Widerlegung von (1d) akzeptieren. Diese Intuition gilt es jetzt zu präzisieren. Man kann dies in einer logischen Rekonstruktion auf verschiedene Arten zu tun versuchen, von denen ich zwei kurz skizzieren will:

2.1 Die statistische oder induktive Lösung

In dem üblichen Allsatzschema

$\forall(\mathrm{x})(\boldsymbol{P}(\mathrm{x}) \rightarrow$ (x) $(\mathrm{x})$

wird das Konditional $\rightarrow$ ersetzt durch ein generisches Konditional $*$, dessen Wahrheitsbedingungen lauten:

$\boldsymbol{p}(\mathrm{x}) * \boldsymbol{p}(\mathrm{x})$

ist wahr gdw. die Zahl der Erfüllungsfälle (d. h. Belegungen für die Individuenvariabeln) für

$\boldsymbol{P}(\mathrm{x}) \wedge \boldsymbol{P z}(\mathrm{x})$

$\mathrm{g} r \ddot{o} B$ e $r$ ist als die für

$\mathfrak{P}(\mathrm{x}) \wedge\left(\neg(\mathfrak{P} \mathbf{x}(\mathrm{x})) .^{13}\right.$

NB.: Für $\multimap$ gilt die Kontraposition $\mathrm{n}$ i $\mathrm{ch} \mathrm{t}$, d.h., aus

$\alpha * \beta$

folgt nicht

$\neg \beta * \neg \alpha$ !

Eine solche Lösung gleicht die Analyse generischer Sätze an die von Frequenzadverbien wie meist, oft etc. an. ${ }^{14}$ Die Frage ist jedoch, ob:

(1h) Die meisten Löwen haben eine Mähne.

als systematische Paraphrase von (1e) gelten kann. Weiter unten werden sich noch weitere Probleme dieser Lösung zeigen.

2.2 Die Redehintergrund-Lösung

Eine zweite Idee besteht darin, daß man bei der Bewertung von Sätzen einen $\mathrm{R}$ e d e h in t e r g r u nd, verstanden als Menge von Sätzen, berücksichtigt - das braucht man ohnehin für eine Analyse von Modalität. ${ }^{15}$

13 Das ist die Grundidee in Link (1988).

14 Vgl. z.B. die Analyse in Cresswell (1973, S. 136).

15 Ausführlich dazu Kratzer (1978) und (1981). 
Sätze werden grundsätzlich relativ zu Redehintergründen (d.h. Mengen

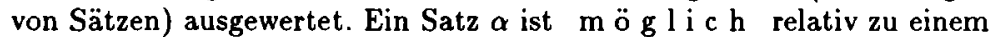
Redehintergrund $R, \diamond_{R}(\alpha)$, gdw. es unter den möglichen Welten, die alle Sätze von $R$ erfüllen $m$ ind e $s$ e $n s$ e i $n$ e gibt, die auch $\alpha$ erfüllt. Der dazu duale Notwendigkeitsoperator $\prod_{R}(\alpha)$ wird wie üblich definiert: [ $]_{\mathbf{R}}(\alpha)$ ist wahr $\mathrm{gdw}$. a 1 l e möglichen Welten, die alle Sätze von $\mathrm{R}$ erfüllen, auch $\alpha$ erfüllen. ${ }^{16}$

Für die Interpretation generischer Sätze kann man nun vorschlagen: ist das Konsequens des generischen Satzes $\alpha \rightarrow \beta$, also $\beta$, überhaupt möglich, d.h., wird seine Wahrheit durch den Redehintergrund nicht als unmöglich ausgewiesen, dann gilt relativ zu diesem Redehintergrund das klassische Konditional. Dies ist eine Präzisierung der eingangs eingeführten Idee, daß generische Interpretationen als „im Prinzip” gültige Allsätze zu behandeln sind. ${ }^{17}$

Damit lassen sich die Wahrheitsbedingungen für ein generisches Konditional $\rightarrow$ folgendermaßen angeben:

$(\alpha \rightarrow \beta)$ ist wahr in der Welt $w_{\circ}$ zu einer Zeit t' relativ zu einem Redehintergrund $\mathbf{R}$ ' $\mathbf{g d w}$. für a ll e Redehintergründe $\mathbf{R}$ und alle Zeiten $t$ gilt: $\square_{\mathrm{R}}\left(\left(\circ_{\mathrm{R}}(\beta) \rightarrow(\alpha \rightarrow \beta)\right)\right){ }^{18}$

Um zu sehen, inwieweit diese Interpretation adäquat ist, betrachten wir die möglichen Wahrheitswertverteilungen in der folgenden Tabelle:

\section{TABELLE}

$\begin{array}{lllllll} & \prod_{\mathbf{R}} & \left(\left(\odot_{\mathbf{R}}(\beta)\right.\right. & \rightarrow & (\alpha & \rightarrow & \beta))) \\ 1 & \mathbf{w} & \mathbf{w} & \mathbf{w} & \mathbf{w} & \mathbf{w} & \mathbf{w} \\ 2 & \mathbf{f}\left(\mathbf{w}^{*}\right) & \mathbf{w} & \mathbf{f} & \mathbf{w} & \mathbf{f} & \mathbf{f} \\ 3 & \mathbf{w} & \mathbf{w} & \mathbf{w} & \mathbf{f} & \mathbf{w} & \mathbf{w} \\ 4 & \mathbf{w} & \mathbf{w} & \mathbf{w} & \mathbf{f} & \mathbf{w} & \mathbf{f} \\ 5 & \mathbf{w} & \mathbf{w}(\mathbf{f} *) & \mathbf{w} & \mathbf{w} & \mathbf{w} & \mathbf{w}\end{array}$

${ }^{16}$ Diese Behandlung der Modalitäten folgt Montague (1960).

17 Von der Grundidee her ähnelt die hier vertretene Theorieskizze den 'Default-Theorien', dazu z.B. Morreau 1988,, Reiter 1980, Strigin 1985 und Thomason 1988, außerdem den Beitrag von Reinhard Blutner in diesem Band. Die nicht-Monotonie ergibt sich hier dadurch, daB die verschiedenen Redehintergünde bezüglich der Möglichkeit des Konsequenz varïeren.

$18 \mathrm{Daß}$ dies für alle Zeiten und alle Redehintergründe als gültig gesetzt wird, ist parallel zu einer Behandlung als „ständiger default"! Siehe dazu Strigin $(1985, \mathrm{~S} .45 \mathrm{ff}$.). $\mathrm{DaB}$ der vorgeschaltete Notwendigkeitsoperator nötig ist, davon haben mich Gisela Zifonun und Helmut Frosch überzeugt. Das Argument ist, daß sonst die Falschheit von $\diamond_{\mathbf{R}} \beta$ die Wahrheit des gesamten Ausdrucks garantieren würde, was intuitiv unangemessen ist. 


\begin{tabular}{|c|c|c|c|c|c|}
\hline 6 & $\mathbf{w}$ & f & $\mathbf{w}$ & $w$ & $f$ \\
\hline 7 & $\mathbf{w}$ & $w(f *)$ & $w$ & $f$ & $\mathbf{w}$ \\
\hline 8 & $\mathbf{w}$ & f & $w$ & f & $w$ \\
\hline 9 & f & $\mathbf{w}$ & $w$ & $w$ & $w$ \\
\hline 10 & f & $\mathbf{w}$ & $f$ & $\mathbf{w}$ & f \\
\hline 11 & $f$ & $\mathbf{w}$ & $\mathbf{w}$ & f & $w$ \\
\hline 12 & f & $\mathbf{w}$ & $w$ & f & $\mathbf{w}$ \\
\hline 13 & f & $\mathbf{w}(f *)$ & $\mathbf{w}$ & $\mathbf{w}$ & $\mathbf{w}$ \\
\hline 14 & f & f & $w$ & $w$ & f \\
\hline 15 & f & $w(f *)$ & $\mathbf{w}$ & f & $\mathbf{w}$ \\
\hline 16 & f & f & $\mathbf{w}$ & f & $\mathbf{w}$ \\
\hline
\end{tabular}

NB.: In den Zeilen 5, 7, 13 und 15 kann in der zweiten Spalte der Wert $f$, der nach einer rein mechanischen Kombinatorik dort zu stehen käme, nicht auftreten, da ja

$\gamma \rightarrow 0(\gamma)$

ein $\mathrm{T}$ h e or e m der Modallogik ist - wenn etwas der Fall ist, dann ist es auch möglich! Ebenso kann in der ersten Spalte in Zeile 2 der Wert w nicht auftreten, da

$(\neg \gamma) \rightarrow(\neg(\square(\gamma))$

ebenfalls ein Theorem ist - was nicht der Fall ist, das ist auch nicht notwendigerweise der Fall! Ohne uns in alle Einzelheiten zu vertiefen können wir folgendes festhalten: Unser abgeschwächtes Konditional $\longrightarrow$ unterscheidet sich vom klassischen Konditional genau dadurch, daß es - in begründeten Einzelfällen - Ausnahmen zuläßt, man sehe Spalte 6! Dies bedeutet, daß eine Formalisierung von (le), $\forall(\mathbf{x})(\mathbf{X}(\mathbf{x}) \longrightarrow \mathbf{S H}(\mathbf{x})), \mathrm{z} . \mathrm{B}$.

auch dann wahr wäre, wenn die Belegung für $x$ ein Individuum zuordnete, das zwar ein Löwe ist, aber keine Mähne hat, weil im Redehintergrund diese Möglichkeit blockiert wird, indem dort z.B. die Information steckt, daß es sich um eine Löwin, einen Junglöwen oder gar um Erich

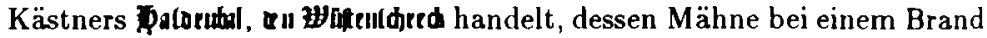
abgesengt wurde und der keine Mähne hat, sondern ein Toupet! ${ }^{19}$ Übrigens paßt diese Lösung auch zu den gesetzesartigen und taxonomischen Sätzen, die wir oben kurz gestreift haben; hier sind die jeweiligen Hinterglieder des generischen Konditionals relativ zum Redehintergrund notwendig - und damit natürlich auch möglich.

$\mathrm{Zu}$ beachten ist, daB Re d e hin t e $\mathbf{r} \mathbf{I} \mathbf{u} \mathbf{n} \mathbf{d}$ ein dynamisches Konzept ist; der Redehintergrund verändert sich im Laufe eines Diskurses durch neu hinzukommende Informationen. Die Fortschreibung von

19 Kästner (1962. S.11). 
Redehintergründen macht Erweiterungen der klassischen Logik in Richtung auf eine Dynamische Semantik nötig, und für ihre Strukturierung kann man sich die Ergebnisse der Kognitionspsychologie, insbesondere Prototypizitätstheorien, zunutze machen. ${ }^{20}$

\section{Generische Interpretation von Nominalphrasen}

Wir kommen damit zu unserer zweiten Hauptfrage - Wie passen die verschiedenen Stiefel auf den einen Leisten? Die generische Interpretation von Sätzen hängt wesentlich ab von der Interpretation der in ihnen stehenden Nominalphrasen. Wir haben in Sätzen mit generischer Interpretation Nominalphrasen mit unbestimmtem oder bestimmtem Artikel, jeweils im Singular und Plural, wir haben artikellose Plurale und artikellose Substanzausdrücke im Singular als verschiedene Stiefel, und wir haben die abgeschwächte Allaussage als den einen, einheitlichen Leisten, über den wir sie jetzt der Reihe nach ziehen wollen.

3.1 Generische Interpretation bei Nominalphrasen mit unbestimmtem Artikel

Um zu zeigen, wie eine generische Interpretation von Sätzen wie:

Ein Löwe hat eine Mähne.

zustandekommt, müssen wir zunächst die Bedeutung des unbestimmten Artikels angeben: der unbestimmte Artikel greift aus den möglichen Denotaten, die die Deutung des Nomens bereitstellt, irgendeines auf als Gegenstand der Prädikation, und er führt es, falls noch nicht vorhanden, als Redegegenstand ein. ${ }^{21}$ Damit gelangen wir zu folgender direkter Interpretation für (12):

$I_{(12)}: \exists(x)(\mathbf{Z}(x) \wedge \mathfrak{A t}(\mathrm{x}))$

(12) wird also wahr, wenn es mindestens ein Individuum gibt, das Löwe ist und eine Mähne hat. An dieser Interpretation kann nun in bestimmten Kontexten, insbesondere in solchen, wo weder im Vor-noch im Nachtext von Löwen wen die Rede ist, eine Uminterpretation ansetzen durch ein

20 Eine solche Logik schlagen z.B. Groenendijk/Strokhof (1991) vor.

21 Das ist die klassische Analyse als Existenzquantor. Für eine ausführliche Diskussion sehe man Heim (1991, Absatz 2). 


\section{RÄSONNEMENT A'LA GRICE 22}

Das läßt sich folgendermaßen skizzieren:

A) Ich habe keinen Hinweis darauf, daß der Sprecher gegen die Maximen „Sag nur Relevantes!” oder „Sag nur Wahres!” verstößt.

B) Wörtlich interpretiert ist (12) kein relevanter Beitrag, da weder eine Prädikation über ein bereits eingeführtes Individuum noch die Einführung eines neuen vorliegt. Die Auswahl eines geeigneten Individuums ist folglich nicht möglich.

C) Also ist irrelevant, welches Individuum ausgewählt wird.

D) Dies kann (wg.A) nur dann sein, wenn (12) für beliebige Individuen aus der Bezugsmenge wahr wird. Also ist der Ubergang zu:

$\mathrm{I}_{(12)}: \forall(\mathrm{x})(\mathbf{x}(\mathrm{x}) \rightarrow$ At $(\mathrm{x}))$ p r a g m a t i s c h gerechtfertigt.

Damit sind wir unserem Leisten „abgeschwächter Allsatz” ein gutes Stück näher gerückt und müssen nur noch die Abschwächung bewerkstelligen.

E) Ich habe keinen Hinweis darauf, daß der Sprecher gegen die Maxime „Mach Deinen Beitrag so informativ wie möglich!” verstößt. Da er weder (12') noch (12") geäußert hat

(12') Alle Löwen haben eine Mähne.

(12") Jeder Löwe hat eine Mähne.

denen $I^{\prime}{ }_{(12)}$ direkt entspricht, bin ich berechtigt, $I_{(12)}$ abzuschwächen zu $\mathrm{I}_{(12)}: \forall(\mathrm{x})(\mathbf{x}(\mathrm{x}) \rightarrow \boldsymbol{H}(\mathrm{x}))$.

Dieses Räsonnement macht das Prinzip generischer Uminterpretation deutlich: Kann für die wörtliche Deutung der zu interpretierenden NP mit unbestimmtem Artikel keine Auswahl getroffen werden, „äuft die Artikelbedeutung leer”, (Schritt B), so wird ein pragmatischer Umstieg über die Allsatzbedeutung zur generischen Lesart ausgelöst (Schritte C,E). Würde man übrigens für das Beispiel (12) statt des hintergründigen das statistische Konditional verwenden, so würde der Satz schlicht falsch - es gibt nämlich mehr Löwinnen als Löwen. Versucht man das dadurch zu vermeiden, daß man postuliert, im Vorderglied des generischen, statistischen Konditionals $*$ müsse das Prädikat auf ein solches beschränkt werden, das eine kon t ext uell erkennbare homogene $S$ ubklasse aus der Extension des ursprünglichen Prädikats denotiert, so sitzt man in der selben Tinte wie oben die Prototyp-einfach-Semantik bei

22 Siehe dazu Grice (1975) und Posner (1979). 
(1g) Der Löwe bringt lebende Junge zur Welt. ${ }^{23}$

Nichts scheint hier gegen einen Redehintergrund zu sprechen, der die Information enthält, daß Löwinnen keine Mähne haben und männliche Löwen keine Jungen zur Welt bringen, wodurch die unerwünschten Schlüsse jeweils blockiert werden, die intuitiv angemessenen jedoch nicht.

3.2 Generische Interpretation bei Nominalphrasen mit bestimmtem Artikel

Die Bedeutung des bestimmten Artikels kann zunächst dahingehend bestimmt werden, daB eine NP mit bestimmtem Artikel das salienteste Element aus dem Bereich der möglichern Nominalphrasendenotate bezeichnet, o d e r, falls im Kontext kein salientestes Element identifizierbar ist, als Gattungsbezeichnung interpretiert wird, sofern dies möglich ist. Der Bereich möglicher Nominalphrasendenotate ist für singularische NP einfach die entsprechende Menge, für pluralische NP ist es ein Konstrukt, das alle möglichen Summenobjekte oder Kollektive der entsprechenden Individuen umfaßt, und für Substanz-NP ist es ein Konstrukt, das alle möglichen Portionen der entsprechenden Substanz bereitstellt. ${ }^{24}$

Für die Beispiele

(13a) Der Löwe hat eine Mähne.

(13b) Die Löwen haben eine Mähne.

ergibt sich: der erste Beispielsatz erhält folgende direkte Interpretation: $I_{(13 a)}$ :

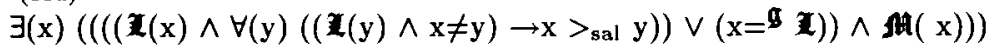

Ist kein salientester Löwe vorhanden, so ergibt sich:

$I^{\prime}(13 a): f(S)$

(\$ $\mathbf{I}$ bezeichne die Gattung Löwe).

Da aber Gattungen für alle Zeitpunkte als die Menge der zum jeweiligen Zeitpunkt existierenden Summen von entsprechenden Individuen rekonstruiert werden können, so gilt:

$\boldsymbol{x}=\lambda \mathbf{t}(\lambda \mathbf{x}(* \mathbf{z}(\mathbf{x})))$

23 Vgl. Link (1988, S. 317). Im übrigen muß man fairerweise einräumen, daß in "normalen” Fällen bei der induktiven Variante dieselben Sätze wahr werden wie bei der hier favorisierten Redehintergrundslösung.

24 Das ist im Prinzip die klassische Russell'sche Analyse, erweitert durch die Salienzidee von David Lewis und generalisiert für Plural- und Substanz-NP. Siehe Lewis (1979). 
also an jedem Auswertungszeitpunkt $\mathrm{t}^{\mathrm{t}}$ :

$\lambda \mathbf{x}(\boldsymbol{*} \mathbf{\mathbf { X }}(\mathrm{x}))$;

somit ergibt sich durch Einsetzen:

$I^{\prime \prime}{ }_{(13 a)}: \operatorname{Att}(\lambda x(* \mathbf{X}(\mathrm{x})))$.

(NB.: * ist das entsprechende P l u r a $]$ p r ä d i k a t, dessen Denotatbereich alle Summen von Einzelindividuen umfaßt.) ${ }^{25}$

$\operatorname{Da} \lambda x(* \mathbf{x}(\mathrm{x}))$ die Menge a ll e r Summen von Löwen bezeichnet, folgt daraus wegen der Distributivität von

$\mathbf{I}^{\text {“" }}(13 \mathbf{a}) \forall(\mathbf{x})(\boldsymbol{*} \mathbf{X}(\mathbf{x}) \rightarrow \boldsymbol{H A}(\mathbf{x}))$;

da zur Extension von $\boldsymbol{*} \mathbf{x}$ auch die maximale Summe von Löwen gehört folgt daraus, wiederum wegen der Distributivität von ft:

$\mathrm{I}^{\text {"“ }}(13 \mathrm{a}): \forall(\mathrm{x})(\mathbf{X}(\mathrm{x}) \rightarrow \mathbf{S H}(\mathrm{x}))$.

Dies kann dann wie oben abgeschwächt werden!

Für (13b) läßt sich eine ähnliche Überlegung anstellen, die in Ermangelung eines salientesten Summenobjekts für die direkte Interpretation von

$\mathrm{I}_{(13 \mathrm{~b})}: \exists(\mathrm{x})\left(* \mathbf{x}(\mathrm{x}) \wedge \forall(\mathrm{y})\left((* \mathbf{x}(\mathrm{y}) \wedge \mathrm{x} \neq \mathrm{y}) \rightarrow \mathrm{x}>>_{\mathrm{sal}} \mathrm{y}\right) \wedge \boldsymbol{\wedge t}(\mathrm{x})\right)$

das maximale auswählt, wonach dann wie bei (13a) weiter geschlossen werden kann. Die Wahrheitsbedingungen für die generische Interpretation von $(13 \mathrm{a}, \mathrm{b})$ können somit intuitiv so formuliert werden: Diese Sätze sind jeweils wahr $\mathrm{gdw}$. für alle $\mathrm{x}$ gilt: ist $\mathrm{x}$ ein Löwe, und enthält der Redehintergrund keine Informationen, die das unmöglich machen, so hat $x$ eine Mähne. Im Gegensatz zu der Analyse von Sätzen mit unbestimmtem Artikel konnte hier ein Großteil der notwendigen Schritte bei der Ableitung der generischen Interpretation in de $\mathrm{r} S$ e m a n t i $k$ getan werden, falls man die von Link vorgeschlagene Pluralanalyse akzeptiert. Nur die Abschwächung des Allsatzes geht noch griceanisch vor sich.

\subsection{Generische Interpretation bei Nominalphrasen ohne Artikel}

Bei den entsprechenden Nominalphrasen handelt es sich entweder um bloße Plurale, oder urn Substanzbezeichnungen im Singuar. Deshalb wählen wir den Beispielsatz

Katzen würden Whiskas kaufen.

in dem beide Arten von Ausdrücken auftreten.

Artikellose NP werden, wie schon in der traditionellen Semantik üblich, analog zu NP mit unbestimmtem Artikel behandelt, d.h., ihr Denotat

25 Für eine detaillierte Darstellung der Probleme sehe man die in Anm. 12 genannte Literatur. 
wird aus dem jeweiligen Denotatbereich ausgewählt, den das artikellose Nomen vorgibt; dabei ist gleichgültig, ob dies ein Pluralnomen oder eine Substanzbezeichnung ist. Als wörtliche Bedeutung ergibt sich für das Beispiel damit

$\mathrm{I}_{(14)} \exists(\mathrm{x})\left(* \mathbf{z}(\mathrm{x}) \wedge \exists(\mathrm{y})\left({ }^{\mathfrak{l}} \boldsymbol{\boldsymbol { \Psi }}(\mathrm{y}) \wedge \boldsymbol{w} \mathbf{z}(\mathrm{x}, \mathrm{y})\right)\right)$

(NB.: $\mathfrak{L}_{\mathfrak{F}}$ ist ein Substanzprädikat, dessen Denotatbereich aus allen Quantitäten der entsprechenden Substanz besteht.) ${ }^{26}$

Ähnlich wie beim unbestimmten Artikel kann man über im Kontext fehlende Kriterien für die Auswahl geeigneter Summenobjekte und Substanzportionen per Räsonnement zu einem entsprechenden Allsatz über alle Summen von Katzen und alle Portionen von Whiskas gelangen:

$\forall(\mathbf{x})\left(* \mathbf{z}(\mathbf{x}) \rightarrow \forall(\mathbf{y})\left({ }^{\mathfrak{l}} \boldsymbol{z}(\mathrm{y}) \rightarrow \boldsymbol{H} \mathbf{z}(\mathbf{x}, y)\right)\right)$

Wegen der Distributivität von $\boldsymbol{\Psi}$ gelangt man auch zu dem Allsatz über einzelne Individuen und per Abschwächung zu den generischen Lesarten:

$\forall(\mathbf{x})(* \mathbf{z}(\mathbf{x}) \rightarrow \forall(\mathbf{y})(\boldsymbol{l} \boldsymbol{z}(\mathbf{y}) \rightarrow \boldsymbol{w} \mathbf{z}(\mathbf{x}, \mathbf{y})))$

für Katzenkollektive bzw.

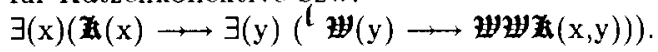

für einzelne Individuen.

Die Wahrheitsbedingungen für 14 wären also: Für alle $\mathrm{x}$ und y gilt: wenn $\mathrm{x}$ eine beliebige Summe von Katzen ist (bzw. eine beliebige Einzelkatze) und y eine beliebige Substanzportion Whiskas, und wenn der Redehintergrund dies nicht unmöglich macht, dann würde $\mathrm{x}$ auch y kaufen. Durch den Redehintergrund würde dann dafür Sorge getragen, daß nur geeignet große bzw. kleine Portionen von Whiskas und nur geeignet kaufkräftige Katzen bzw. Katzenkollektive in Betracht gezogen werden.

Bei der Behandlung von Substanzausdrücken stößt übrigens die statistische Lösung, wie auch die Prototyp-einfach-Lösung, auf Schwierigkeiten. Das zeigt sich an Sätzen wie

Bier ist bekömmlich.

denn natürlich gibt es sehr viel mehr Substanzportionen von Bier, deren Genuß dem Menschen nicht bekommt, als solche, deren Genuß ihm frommt.

In der favorisierten Hintergrundslösung kann man diese aber ausschließen, indem man über zivilisatorische Konventionen die Menge der zu beachtenden Bierportionen einschränkt.

${ }^{26}$ Dies folgt wieder Link (1983). 


\section{Schlußbemerkung}

Nach der Bestimmung des eigentlichen Problems - distributive Prädikation über gattungsbezeichnende Nomina - haben wir zunächst eine semantische Interpretation für ein generisches Konditional entwickelt, die im Rahmen klassischer Modallogik formuliert werden konnte. Danach haben wir gezeigt, wie durch semantische Eigenschaften von Nominalphrasen, die in einer durch die Link'schen Analysen für Plurale und Substanzausdrücke erweiterten logischen Semantik beschreibbar sind, sowie durch griceanische Räsonnements eine Uminterpretation von wörtlichen zu generischen Bedeutungen geleistet werden kann.

Wichtig scheint mir der Hinweis, daß es nicht nötig war, neue Konzepte für die generische Interpretation in die Theorie einzuführen; eine Variante des Möglichkeitsbegriffs, die von Link vorgeschlagene Analyse von Pluralen und Substanzausdrücken und griceanische Räsonnements ermöglichte die Analyse der generischen Sätze.

Die Herausforderung an die Prototypensemantik liegt darin, daß m.E. das sogenannte "Begrenzungsproblem" in der Wortsemantik, das angeblich nicht im klassischen Rahmen lösbar ist, gelöst werden kann, wenn man außer den "harten” Bedeutungspostulaten (mit $\rightarrow$ formuliert) noch "weiche" zur Verfügung hat (mit $\longrightarrow$ zu formulieren). Ergebnisse der Kognitionspsychologie können im Bereich einer realistischen Rekonstruktion von Redehintergründen eine wichtige Rolle spielen, aber der Prototypen s e $\mathrm{m}$ a $\mathrm{n} \mathrm{t}$ i k käme ihr antilogizistischer Impetus abhanden!

\section{Literatur}

Blutner, Reinhard (1988): Defaults and the Meaning of Generic Sentences, in: Krifka, M. (Hg.) S. 3-30.

Bāuerle, Rainer/Egli, Urs/v.Stechow, Arnim (Hg.) (1979): Semantics from different points of view, Berlin, Heidelberg, New York.

Cresswell, Max (1973): Logics and Languages, London.

Cole, P./Morgan, J.L. (Hg.) (1975): Syntax and Semantics, vol. 3: Speech Acts, New York.

Davidson, Donald/Harman, Gilbert (Hg.) (1972): Semantics of Natural Language, Dordrecht.

Eisenberg, Peter (1986): Grundriss der deutschen Grammatik, Stuttgart.

Eikmeyer, H.J./Rieser, H. (Hg.) (1981): Words, Worlds and Contexts, Berlin.

Grewendorf, G. (Hg.) (1979): Sprechakttheorie und Semantik, Frankfurt am Main.

Grice, Herbert Paul (Hg.) (1975): Logic and Conversation, in Cole, P./Morgan, J.L., S. 41-58. 
Groenendijk, Jeroen \& Stokhof, Martin (1991): Dynamic Predicate Logic, Linguistics and Philosophy 14, S. 39-100.

Heim, Irene (1991): Artikel und Definitheit, in: v.Stechow, A./Wunderlich, D., S.487-535.

Kästner, Erich (1962): Die Konferenz der Tiere, Berlin.

Kratzer, Angelika (1978): Semantik der Rede. Kontexttheorie, Modalwörter, Konditionalsätze, Kōnigstein.

Kratzer, Angelika (Hg.) (1981): The Notional Category of Modality, in: Eikmeyer, H.J./Rieser, H., S. 38-74:

Krifka, Manfred, (Hg.) (1987): (unter Mitarbeit von Claudia Gerstner) An Outline of Genericity, SNS - Bericht, Tübingen.

Krifka, Manfred (Hg.) (1988): Genericity in Natural Language, SNS-Bericht 88-42, Tübingen.

Krifka, Manfred (1991): Massennomina, in: v. Stechow, A./Wunderlich, D., S. 399-417.

Langacker, Ronald: (1987): Foundations of Cognitive Grammar, Stanford.

Lewis, David (General Semantics, in: Davidson,D./Harman, G. (Hg.), S. 169218.

Lewis, David (1983): Scorekeeping in a Language Game, in: Bäuerle et al. (Hg.), S. 172-187.

Link, Godehard (1983): The Logical Analysis of Plurals and Mass Terms: A Lattice - Theoretical Approach, in Bäuerle et al. (Hg.) S. 302-323

Link, Godehard (1988): Dependency in the Theory of Generics, in Krifka, S. 313-335.

Link, Godehard: Plural, in v. Stechow, A./ Wunderlich, D., S. 418-440.

Montague, Richard (1960): Logical Necessity, Physical Necessity, Ethics, and Quantifiers, in: Inquiry 4, S. 259-269.

Morreau, Michael (1988): Default Formalisms for Generics: A Consumer Report, in: Krifka, S. 337-356.

Posner, Roland (1979): Bedeutung und Gebrauch der Satzverknüpfer in den natürlichen Sprachen, in: Grewendorf, G. (Hrsg.) S. 345-385.

Reiter, Richard (1980): A Logic for Default Reasoning, Artificial Intelligence 13.1, S. 81-132.

Roth, Eugen (1956): Eugen Roths Kleines Tierleben, München.

Stechow, A.v./Wunderlich, D. (Hg.) (1991) Semantik. Ein internationales Handbuch der zeitgenössischen Forschung, Berlin, New York.

Strigin, Anatoli (1985): Eine Semantik für generische Sätze, in: Linguistische Studien, Reihe A, 125, Generische Sätze, Prototypen und Defaults, Berlin.

Swift, Johnathan (992): Gullivers Travels, 1726, Hertfordshire (Wordsworth Classics).

Taylor, John R., Schemas, Prototypes, and Models: in Search of the Unity of the Sign, in: Tzohatzidis, S.L. (Hg.), S. 521-534. 
Thomason, Richmond (1988): Theories of Nonmonotonicity and Natural Language Generics, in: Krifka, M. (Hg.) S. 395-406.

Tzohatsidis, Savas L. (Hg.) (1989): Meanings and Prototypes, London, New York. 